\title{
Vertical Cost-Information Sharing in a Food Supply Chain with Multiple Unreliable Suppliers and Two Manufacturers
}

\author{
Junjian Wu, ${ }^{1}$ Haiyan Wang, ${ }^{1}$ and Henry $\mathrm{Xu}^{2}$ \\ ${ }^{1}$ School of Economics and Management, Southeast University, Nanjing 210096, China \\ ${ }^{2}$ UQ Business School, The University of Queensland, Brisbane, QLD 4072, Australia \\ Correspondence should be addressed to Haiyan Wang; hywang@seu.edu.cn
}

Received 14 September 2017; Accepted 14 November 2017; Published 11 December 2017

Academic Editor: Chunming Shi

Copyright (c) 2017 Junjian Wu et al. This is an open access article distributed under the Creative Commons Attribution License, which permits unrestricted use, distribution, and reproduction in any medium, provided the original work is properly cited.

\begin{abstract}
This paper considers a food supply chain where multiple suppliers provide completely substitutable food products to two manufacturers. Meanwhile, the suppliers face yield uncertainty and the manufacturers face uncertain production costs that are private information. While the suppliers compete on price, the manufacturers compete on quantity. We build a stylized multistage game theoretic model to analyze the issue of vertical cost-information sharing (VCIS) within the supply chain by considering key parameters, including the level of yield uncertainty, two manufacturers' cost correlation, the correlated coefficient of suppliers' yield processes, and the number of suppliers. We study the suppliers' optimal wholesale price and the manufacturers' optimal order quantities under different VCIS strategies. Finally, through numerical analyses, we examine how key parameters affect the value of VCIS to each supplier and each manufacturer, respectively. We found that the manufacturers are willing to share cost information with suppliers only when the two manufacturers' cost correlation is less than a threshold. While a high correlated coefficient of suppliers' yield processes and a large number of suppliers promote complete information sharing, a high level of yield uncertainty hinders complete information sharing. All these findings have important implications to industry practices.
\end{abstract}

\section{Introduction}

Information asymmetry between supply chain (SC) members is a great challenge for food SC management. Information sharing is an effect tool to eliminate the impact of information asymmetry on SC partners' performances [1]. Demand information sharing [2] and cost-information sharing [3] have been much discussed by scholars. Most of literature assumed that the suppliers are reliable, while in industry practices the suppliers are unreliable due to various uncontrollable factors including equipment error and natural hazards. Our work focuses on vertical cost-information sharing by considering the suppliers with yield uncertainty.

Yield uncertainty is a popular phenomenon in the agricultural and food industries. The quality of agricultural and food products is very sensitive to temperature, humidity, and other natural conditions. For example, in agribusinesses, the yield of crop per acre is uncertain as it depends on such factors as climate condition, irrigation level, and so forth (http://nca2014.globalchange.gov/report/sectors/agriculture). Recently Recha et al. [4] have provided a symmetric report about the effect of climate-correlated conditions on food quality. Some scholars analyzed food SC management in different views. For example, Nyamah et al. [5] and MacKenzie and Apte [6] investigated food quality risk management from the operations management perspective. Keizer et al. [7] and Jin et al. [8] investigated food SC by considering supply network and food traceability, respectively. They examined food SC in a complete information situation, whereas in reality information asymmetry between upstream and downstream SC firms often exists.

After the sourcing firms (i.e., manufacturers) have received the product from farmers (i.e., suppliers), they process these products into final products with one unit marginal cost and sell in a common market. The marginal costs are also uncertain due to uncertain labor cost, uncertain storage costs, and other uncontrollable factors. Both supply uncertainty and cost uncertainty directly affect upstream and 
downstream SC firms' wholesale prices and order quantity decisions. In this paper, we consider that two manufacturers' costs are their private information.

Motivated by the real business situation as mentioned above, this paper examines a SC with multiple suppliers and two manufacturers and aims to answer two important questions. First, how do suppliers and manufacturers make decisions on wholesale prices and order quantities, respectively, under different VCIS scenarios? (2) Are manufacturers willing to disclose cost information to suppliers?

To answer the above questions, we build a classic threestage game model. At the 1st-stage VCIS game, each manufacturer has two VCIS strategies: share and not share. Hence, there are four VCIS scenarios: (1) both manufacturers share cost information (i.e., complete cost-information sharing, CCIS); (2) none of them share cost information (i.e., no costinformation sharing, NCIS); (3) manufacturer 1 shares cost information, but manufacturer 2 does not; (4) manufacturer 2 shares cost information, but manufacturer 1 does not. The 2nd stage is the multiple suppliers' selling price game, and the 3rd stage is the two manufacturers' selling quantities game. After solving each subgame, we obtain the suppliers' optimal wholesale prices and the two manufacturers' optimal order quantities under each VCIS scenario. Further, we found that both manufacturers are willing to share cost information with their suppliers. Moreover, we found that complete information sharing will benefit all SC partners and the whole SC.

The remainder of this paper is as follows. Section 2 summarizes the related work. The model framework is shown in Section 3. The equilibrium solutions for four VCIS scenarios are provided in Section 4 . The VCIS game is analyzed in Section 5. Section 6 provides a numerical analysis. Conclusions are drawn in Section 7.

\section{Related Work}

Our research is closely related to information sharing. The literature in this area can be divided into two streams: horizontal information sharing (HIS) and vertical information sharing (VIS) [9].

Some scholars focus on HIS. For example, Clarke [10] and Galor [11] studied HIS in an oligopoly model and showed that no HIS is a unique equilibrium. Kirby [12] investigated the incentive for HIS in an oligopoly model where firms have nonlinear product costs and showed that HIS exists under some conditions. Vives [13] discussed firms' HIS under Cournot competition and Bertrand competition, respectively. $\mathrm{Li}$ [14] examined the inventive for demand and cost-information sharing. Zhu [3] and Zhou and Zhu [15] investigated the incentive for cost-information sharing in a business-to-business setting under Cournot competition. Wu et al. [16] examined HIS by considering firms with capacity constraints. Natarajan et al. [17] analyzed HIS by considering time as an important factor in their model. Jiang and Hao [18] showed that information sharing and cooperative price for firms are strategic complements.

On the other hand, some researchers focus on VIS in a SC context. Li [19] and Zhang [20] took information leakage into account when studying VIS within a SC. Subsequently, Anand and Goyal [21], Kong et al. [22], and Shamir [23] investigated VIS by taking into account the fact that information leakage comes from the upstream suppliers. Li and Zhang [2] examined how the level of confidentiality influences firms' VIS decisions. Ha et al. [24, 25] studied VIS in two competing SCs. Wu et al. [26] examined the relationship between channel construction and VIS. Jiang and Hao [27] examined VIS under different channel structures. Subsequently, Zhou et al. [28] explored the effect of group purchasing organizations (GPOs) on VIS. Zhang and Xiong [29] explored VIS in a closed-loop SC. These papers focused on demand information sharing. Cost-information sharing has also been researched by several scholars. For example, Yao et al. [30] explored cost-information sharing in a SC by considering value-added costs as retailers' private information. Liu et al. [31] examined the interplay between VCIS and channel choices. Kostamis and Duenyas [32] investigated the value of both cost and demand information sharing. Moreover, Cachon and Lariviere [33], Eksoz et al. [34], and ResendeFilho and Hurley [35] explored the value of information to SC operational decisions.

Our work is different from those works above in that we examine the incentive for VCIS within a SC which has $n$-suppliers and two competing manufactures. The suppliers are subject to yield uncertainty and are engaged in setting price. Therefore, this is the first study to address the abovementioned business scenario.

\section{Model Framework}

In this paper, we consider a SC with $n$ unreliable suppliers and two competing manufacturers with private cost information. The suppliers sell complete substitutable food products to the two manufacturers.

3.1. Supply and Cost-Information Structures. The suppliers' yield uncertainty is modeled as a random proportion [36]; that is, if one manufacture's order quantity for each supplier is $q_{i k}$, the final quantity received from each supplier is a random proportion $y_{k}$ of $q_{i k}$, that is, $y_{k} q_{i k}$. We assume that $y_{k} \in(0,1]$ and $E\left[y_{k}\right]=\mu \leq 1$ and $\operatorname{Var}\left[y_{k}\right]=\sigma_{y}^{2}$ (the same assumption is provided in [37-39]). Also, we assume that $\rho=\operatorname{Cov}\left(y_{k}, y_{l}\right) / \sigma_{y}^{2}$ and $\rho \in[-1,1), k \neq l$. In addition, let $\delta=\sigma_{y} / \mu$ denote the level of yield uncertainty. Each supplier's expected cost is $c$, where $c=c_{1} / \mu+c_{2}$ and $c_{1}$ and $c_{2}$ represent the unit manufacture cost and the unit transport cost, respectively.

Each manufacturer's marginal cost $c_{m_{i}}$ is uncertain, and we assume that $c_{m_{i}}$ follows a normal distribution with $E\left[c_{m_{i}}\right]=0$ and $\operatorname{Var}\left[c_{m_{i}}\right]=\sigma^{2}$ [19]. It is also assumed that $c_{m_{i}}$ and $c_{m_{j}}$ satisfy the following: (1) $E\left[c_{m_{i}} \mid c_{m_{j}}\right]=\gamma_{i}+\gamma_{j} c_{m_{j}}$, where $\gamma_{i}, \gamma_{j}$ are positive constants for all $j=3-i, i=1,2$, and (2) $c_{m_{i}}$ and $c_{m_{j}}$ are identically distributed. Therefore, we have

$$
\begin{aligned}
E\left[c_{m_{i}} \mid c_{m_{j}}\right] & =\eta c_{m_{j}} ; \\
E\left[c_{m_{i}} c_{m_{j}}\right] & =\eta \sigma^{2} ;
\end{aligned}
$$




$$
E\left[c_{m_{i}} c_{m_{i}}\right]=\sigma^{2}
$$

where $\eta=\operatorname{Cov}\left[c_{m_{i}} c_{m_{j}}\right] / \sigma^{2}$ and $\eta \in(0,1)$.

3.2. The Demand Function. Similar to $[19,40]$, we assume that the inverse demand function is

$$
P=a-Q_{i}-Q_{j}, \quad j=3-i, i=1,2,
$$

where $a$ is demand intercept, $P$ is the product's retail price, and $Q_{i}$ and $Q_{j}$ are the manufacturer $i$ 's and the manufacturer $j$ 's selling quantities in the common market.

3.3. Sequence Decisions Made by the Manufacturers and Suppliers. The sequence of decisions made by SC members is specified as follows:

(1) Each downstream SC member (i.e., manufacturer) decides whether to share the cost information with upstream SC members (i.e., suppliers) or not.

(2) The suppliers make a decision on prices.

(3) The manufacturers make a decision on selling quantities.

(4) The suppliers make production decisions and transport products.
(5) The yield and cost uncertainties are realized, and the demand is satisfied.

This is a three-stage game problem based on the above sequence. The 1st-stage game is VCIS game. Let $Z_{i}=Y\left(Z_{i}=\right.$ $N$ ) which means that manufacturer $i$ shares (or does not share) cost information with each supplier. Thus, there exists four possible VCIS scenarios in the 1st-stage game: $(Y, Y)$, $(N, N),(Y, N)$, and $(N, Y)$. The other two games are the selling price game for the suppliers and the selling quantity game for the manufacturers.

The main variables that will be used in the paper are summarized in "Notation for Variables" section.

\section{Equilibrium Solutions}

In this section, because strategies $(Y, N)$ and $(N, Y)$ are symmetrical, we only consider strategy $(Y, N)$. We address the manufacturers' optimal decisions $q_{i k}^{*}$ and suppliers' optimal decisions $w_{k}^{*}$ under three possible VCIS scenarios: $(Y, Y)$, $(N, N)$, and $(Y, N)$.

4.1. Subgame $(Y, Y)$ : Both Manufacturers Share Cost Information. If both manufacturers choose VCIS, the suppliers can make an optimal decision based on $c_{m_{1}}$ and $c_{m_{2}}$. One manufacturer can infer the other manufacturer's private cost information from $w_{k}^{Y Y *}$ [19].

Therefore, under subgame $(Y, Y)$, manufacturer $i$ 's $(i=$ $1,2)$ optimization problem is

$$
\max _{q_{i 1}, q_{i 1}, \ldots, q_{i n}} E\left[\pi_{m_{i}} \mid c_{m_{i}}, c_{m_{j}}\right]=E\left\{\left(a-\sum_{i=1}^{2} \sum_{k=1}^{n} y_{k} q_{i k}\right) \sum_{k=1}^{n} y_{k} q_{i k}-\sum_{k=1}^{n} w_{k} y_{k} q_{i k}-c_{m_{i}} \sum_{k=1}^{n} y_{k} q_{i k} \mid c_{m_{i}}, c_{m_{j}}\right\} .
$$

Supplier $k$ 's $(k=1,2, \ldots, n)$ optimization problem is

$$
\begin{aligned}
\max _{w_{k}} & {\left[\pi_{s_{k}} \mid c_{m_{1}}, c_{m_{2}}\right] } \\
& =E\left[\left(w_{k}-c\right) y_{k}\left(q_{1 k}+q_{2 k}\right) \mid c_{m_{1}}, c_{m_{2}}\right] .
\end{aligned}
$$

$q_{i k}^{Y{ }^{*}}(i=1,2, k=1,2, \ldots, n)$ should satisfy the following 1st-order condition:

$$
\begin{aligned}
& q_{i k}^{Y Y *}=\frac{\left(a-w_{k}\right)}{2 \mu(1-\rho) \delta_{y}^{2}} \\
& \quad-\frac{\left(1+\rho \delta_{y}^{2}\right) \sum_{k=1}^{n}\left(a-w_{k}\right)}{2 \mu(1-\rho) \delta_{y}^{2}\left[\left(1+\delta_{y}^{2}\right)+(n-1)\left(1+\rho \delta_{y}^{2}\right)\right]} \\
& \quad-\frac{1}{2 \mu\left[\left(1+\delta_{y}^{2}\right)+(n-1)\left(1+\rho \delta_{y}^{2}\right)\right]} \\
& \quad \cdot E\left(c_{m_{i}} \mid c_{m_{i}}, c_{m_{j}}\right)-\frac{1}{2} E\left(q_{j k}^{Y Y *} \mid c_{m_{i}}, c_{m_{j}}\right) .
\end{aligned}
$$

By Proposition 1 in [14], the unique equilibrium solutions for the manufacturers are specified as

$$
\begin{aligned}
q_{i k}^{Y Y *} & \\
= & \frac{\left(a-w_{k}\right)}{3 \mu(1-\rho) \delta_{y}^{2}} \\
& -\frac{\left(1+\rho \delta_{y}^{2}\right) \sum_{k=1}^{n}\left(a-w_{k}\right)}{3 \mu(1-\rho) \delta_{y}^{2}\left[\left(1+\delta_{y}^{2}\right)+(n-1)\left(1+\rho \delta_{y}^{2}\right)\right]} \\
& -\frac{2}{3 \mu\left[\left(1+\delta_{y}^{2}\right)+(n-1)\left(1+\rho \delta_{y}^{2}\right)\right]} c_{m_{i}} \\
& +\frac{1}{3 \mu\left[\left(1+\delta_{y}^{2}\right)+(n-1)\left(1+\rho \delta_{y}^{2}\right)\right]} c_{m_{j}} .
\end{aligned}
$$

By inserting $q_{i k}^{Y Y *}$ into (4), we obtain $w_{k}^{Y Y *}(k=$ $1,2, \ldots, n)$ which should satisfy the following 1st-order condition: 


$$
\begin{aligned}
& w_{k}^{Y Y *} \\
& =\frac{a+c}{2}-\frac{\left(1+\rho \delta_{y}^{2}\right) \sum_{l \neq k}^{n}\left[a-E\left(w_{l} \mid c_{m_{1}}, c_{m_{2}}\right)\right]}{2\left[(1-\rho) \delta_{y}^{2}+(n-1)\left(1+\rho \delta_{y}^{2}\right)\right]} \\
& \quad-\frac{(1-\rho) \delta_{y}^{2}}{4\left[(1-\rho) \delta_{y}^{2}+(n-1)\left(1+\rho \delta_{y}^{2}\right)\right]}\left(c_{m_{1}}+c_{m_{2}}\right) .
\end{aligned}
$$

Based on Proposition 1 in [14], there exist unique equilibrium solutions $w_{k}^{Y Y *}$. By substituting $w_{k}^{Y{ }^{*}}$ into (5) and simplifying, we obtain Proposition 1.

Proposition 1. The equilibrium solutions for subgame $(Y, Y)$ are specified as follows.

(1) The optimal decisions for the suppliers at equilibrium are

$$
w_{k}^{Y Y *}=\bar{w}_{k}+\xi_{k 1}^{Y Y} c_{m_{1}}+\xi_{k 2}^{Y Y} c_{m_{2}}
$$

where

$$
\begin{aligned}
& \bar{w}_{k}=\frac{(1-\rho) \delta_{y}^{2} a+\left[(1-\rho) \delta_{y}^{2}+(n-1)\left(1+\rho \delta_{y}^{2}\right)\right] c}{\left[2(1-\rho) \delta_{y}^{2}+(n-1)\left(1+\rho \delta_{y}^{2}\right)\right]}, \\
& \xi_{k 1}^{Y Y}=\xi_{k 2}^{Y Y}=-\frac{(1-\rho) \delta_{y}^{2}}{2\left[2(1-\rho) \delta_{y}^{2}+(n-1)\left(1+\rho \delta_{y}^{2}\right)\right]} .
\end{aligned}
$$

(2) The optimal decisions for the manufacturers at equilibrium are

$$
q_{i k}^{Y Y *}=\bar{q}_{i k}+f_{i k i}^{Y Y} c_{m_{i}}+f_{i k j}^{Y Y} c_{m_{j}}
$$

where

$$
\begin{aligned}
\bar{q}_{i k} & =\frac{\left[(1-\rho) \delta_{y}^{2}+(n-1)\left(1+\rho \delta_{y}^{2}\right)\right](a-c)}{3 \mu\left[\left(1+\delta_{y}^{2}\right)+(n-1)\left(1+\rho \delta_{y}^{2}\right)\right]\left[2(1-\rho) \delta_{y}^{2}+(n-1)\left(1+\rho \delta_{y}^{2}\right)\right]}, \\
f_{i k i}^{Y Y} & =-\frac{\left[7(1-\rho) \delta_{y}^{2}+4(n-1)\left(1+\rho \delta_{y}^{2}\right)\right]}{6 \mu\left[\left(1+\delta_{y}^{2}\right)+(n-1)\left(1+\rho \delta_{y}^{2}\right)\right]\left[2(1-\rho) \delta_{y}^{2}+(n-1)\left(1+\rho \delta_{y}^{2}\right)\right]}, \\
f_{i k j}^{Y Y} & =\frac{\left[5(1-\rho) \delta_{y}^{2}+2(n-1)\left(1+\rho \delta_{y}^{2}\right)\right]}{6 \mu\left[\left(1+\delta_{y}^{2}\right)+(n-1)\left(1+\rho \delta_{y}^{2}\right)\right]\left[2(1-\rho) \delta_{y}^{2}+(n-1)\left(1+\rho \delta_{y}^{2}\right)\right]} .
\end{aligned}
$$

In Proposition 1, both $w_{k}^{Y Y *}$ and $q_{i k}^{Y Y *}$ are composed of two parts: one is not dependent on $c_{m_{i}}$ and $c_{m_{j}}$ (i.e., $\bar{w}_{k}$ and $\bar{q}_{i k}$ ), while the other is dependent on $c_{m_{i}}$ and $c_{m_{j}}$ (i.e., $\xi_{k 1}^{Y Y} c_{m_{1}}+\xi_{k 2}^{Y Y} c_{m_{2}}$ and $f_{i i}^{Y Y} c_{m_{i}}+f_{i j}^{Y Y} c_{m_{j}}$ ). Clearly, $\xi_{k 1}^{Y Y}=\xi_{k 2}^{Y Y}<0$, $f_{i k i}^{Y Y}<0$, and $f_{i k j}^{Y Y}>0 . \xi_{k 1}^{Y Y}=\xi_{k 2}^{Y Y}<0$ means that $w_{k}^{Y Y *}$ is negatively related to $c_{m_{i}}+c_{m_{j}} \cdot f_{i k i}^{Y Y}<0$ means that $q_{i k}^{Y Y *}$ responds negatively to $c_{m_{i}}$, and $f_{i k j}^{Y Y}>0$ shows that $q_{i k}^{Y Y *}$ is positively related to $c_{m_{j}}$.
4.2. Subgame $(N, N)$ : No Manufacturer Shares Cost Information. Suppose that no manufacturer shares private cost information with their suppliers. The suppliers' optimal decisions $w_{k}^{N N *}$ are independent of the manufacturers' cost information. Therefore, one manufacturer cannot infer the other firm's cost information from $w_{k}^{N N *}$ [19].

Therefore, under subgame $(N, N)$, the manufacturer $i$ 's $(i=1,2)$ optimization problem is

$$
\max _{q_{i 1}, q_{i 1}, \ldots, q_{i n}} E\left[\pi_{m_{i}} \mid c_{m_{i}}\right]=E\left\{\left(a-\sum_{i=1}^{2} \sum_{k=1}^{n} y_{k} q_{i k}\right) \sum_{k=1}^{n} y_{k} q_{i k}-\sum_{k=1}^{n} w_{k} y_{k} q_{i k}-c_{m_{i}} \sum_{k=1}^{n} y_{k} q_{i k} \mid c_{m_{i}}\right\} .
$$

Supplier $k$ 's $(k=1,2, \ldots, n)$ optimization problem is

$$
\max _{w_{k}}\left[\pi_{s_{k}}\right]=E\left[\left(w_{k}-c\right) y_{k}\left(q_{1 k}+q_{2 k}\right)\right]
$$

Manufacturer $i$ 's optimal order quantities $q_{i k}^{N N *}(i=$ $1,2, k=1,2, \ldots, n)$ should satisfy the following 1st-order condition:

$$
\begin{aligned}
q_{i k}^{N N *} & \\
= & \frac{\left(a-w_{k}\right)}{2 \mu(1-\rho) \delta_{y}^{2}} \\
& \quad-\frac{\left(1+\rho \delta_{y}^{2}\right) \sum_{k=1}^{n}\left(a-w_{k}\right)}{2 \mu(1-\rho) \delta_{y}^{2}\left[\left(1+\delta_{y}^{2}\right)+(n-1)\left(1+\rho \delta_{y}^{2}\right)\right]}
\end{aligned}
$$




$$
\begin{aligned}
& -\frac{1}{2 \mu\left[\left(1+\delta_{y}^{2}\right)+(n-1)\left(1+\rho \delta_{y}^{2}\right)\right]} E\left(c_{m_{i}} \mid c_{m_{i}}\right) \\
& -\frac{1}{2} E\left(q_{j k}^{N N *} \mid c_{m_{i}}\right) .
\end{aligned}
$$

With reference to Proposition 1 in [14], the unique equilibrium solutions for the manufacturers are specified as

$$
\begin{aligned}
q_{i k}^{N N *} & =\frac{\left(a-w_{k}\right)}{3 \mu(1-\rho) \delta_{y}^{2}} \\
& -\frac{\left(1+\rho \delta_{y}^{2}\right) \sum_{k=1}^{n}\left(a-w_{k}\right)}{3 \mu(1-\rho) \delta_{y}^{2}\left[\left(1+\delta_{y}^{2}\right)+(n-1)\left(1+\rho \delta_{y}^{2}\right)\right]} \\
& -\frac{(1+t)}{\mu\left[\left(1+\delta_{y}^{2}\right)+(n-1)\left(1+\rho \delta_{y}^{2}\right)\right][2(1+t)+1]} \\
& \cdot c_{m_{i}} \cdot
\end{aligned}
$$

By inserting $q_{i k}^{N N *}$ into (13), we can obtain the supplier's optimal wholesale price $w_{k}^{N N *}(k=1,2, \ldots, n)$ which satisfies the following 1st-order condition:

$$
w_{k}^{N N *}=\frac{a+c}{2}-\frac{\left(1+\rho \delta_{y}^{2}\right) \sum_{l \neq k}^{n}\left(a-w_{l}^{N N *}\right)}{2\left[(1-\rho) \delta_{y}^{2}+(n-1)\left(1+\rho \delta_{y}^{2}\right)\right]}
$$

Based on Proposition 1 in [14], there exist unique equilibrium solutions $w_{k}^{N N *}$ for the manufacturers. Substituting $w_{k}^{N N *}$ into (13) and simplifying, we obtain Proposition 2.
Proposition 2. The equilibrium solutions for subgame $(N, N)$ are specified as follows.

(1) The optimal decisions for the suppliers at equilibrium are

$$
w_{k}^{N N *}=\bar{w}_{k} .
$$

(2) The optimal decisions for the manufacturers at equilibrium are

$$
q_{i k}^{N N *}=\bar{q}_{i k}+f_{i k i}^{N N} c_{m_{i}}
$$

where

$$
f_{i k i}^{N N}=-\frac{1}{\mu\left[\left(1+\delta_{y}^{2}\right)+(n-1)\left(1+\rho \delta_{y}^{2}\right)\right](2+\eta)} .
$$

Proposition 2 shows that the suppliers' optimal decisions are independent of the manufacturers' private cost information, and manufacturer is optimal decision depends only on $c_{m_{i}} \cdot f_{i k i}^{N N}<0$ indicates that manufacturer $i$ responds negatively to $c_{m_{i}}$.

4.3. Subgame $(Y, N)$ : Only One Manufacturer Shares Cost Information. Suppose only manufacturer 1 shares cost information $c_{m_{1}}$ with their suppliers. The suppliers set $w_{k}^{S N *}$ based on $c_{m_{1}}$. Then manufacturer 2 can infer manufacturer 1's cost information from $w_{k}^{S N *}[19]$.

Therefore, under subgame $(Y, N)$, manufacturer 1's optimization problem is

$$
\max _{q_{11}, q_{11}, \ldots, q_{1 n}} E\left[\pi_{m_{1}} \mid c_{m_{1}}\right]=E\left\{\left(a-\sum_{i=1}^{2} \sum_{k=1}^{n} y_{k} q_{1 k}\right) \sum_{k=1}^{n} y_{k} q_{1 k}-\sum_{k=1}^{n} w_{k} y_{k} q_{1 k}-c_{m_{1}} \sum_{k=1}^{n} y_{k} q_{1 k} \mid c_{m_{1}}\right\} .
$$

Manufacturer 2's optimization problem is

$$
\max _{q_{21}, q_{21}, \ldots, q_{2 n}} E\left[\pi_{m_{2}} \mid c_{m_{1}}, c_{m_{2}}\right]=E\left\{\left(a-\sum_{i=1}^{2} \sum_{k=1}^{n} y_{k} q_{2 k}\right) \sum_{k=1}^{n} y_{k} q_{2 k}-\sum_{k=1}^{n} w_{k} y_{k} q_{2 k}-c_{m_{2}} \sum_{k=1}^{n} y_{k} q_{2 k} \mid c_{m_{1}}, c_{m_{2}}\right\} .
$$

Supplier $k^{\prime} s(k=1,2, \ldots, n)$ optimization problem is

$$
\max _{w_{k}}\left[\pi_{s_{k}} \mid c_{m_{1}}\right]=E\left[\left(w_{k}-c\right) y_{k}\left(q_{1 k}+q_{2 k}\right) \mid c_{m_{1}}\right] .
$$

The two manufacturers' optimal order quantities $q_{1 k}^{Y N *}$ and $q_{2 k}^{Y N *}(k=1,2, \ldots, n)$ should satisfy the following 1storder condition:

$$
q_{1 k}^{Y N *}=\frac{\left(a-w_{k}\right)}{2 \mu(1-\rho) \delta_{y}^{2}}
$$

$$
\begin{aligned}
& -\frac{\left(1+\rho \delta_{y}^{2}\right) \sum_{k=1}^{n}\left(a-w_{k}\right)}{2 \mu(1-\rho) \delta_{y}^{2}\left[\left(1+\delta_{y}^{2}\right)+(n-1)\left(1+\rho \delta_{y}^{2}\right)\right]} \\
& -\frac{1}{2 \mu\left[\left(1+\delta_{y}^{2}\right)+(n-1)\left(1+\rho \delta_{y}^{2}\right)\right]} E\left(c_{m_{1}} \mid c_{m_{1}}\right) \\
& -\frac{1}{2} E\left(q_{2 k}^{Y N *} \mid c_{m_{1}}\right), \\
& q_{2 k}^{Y N *}=\frac{\left(a-w_{k}\right)}{2 \mu(1-\rho) \delta_{y}^{2}}
\end{aligned}
$$




$$
\begin{aligned}
& -\frac{\left(1+\rho \delta_{y}^{2}\right) \sum_{k=1}^{n}\left(a-w_{k}\right)}{2 \mu(1-\rho) \delta_{y}^{2}\left[\left(1+\delta_{y}^{2}\right)+(n-1)\left(1+\rho \delta_{y}^{2}\right)\right]} \\
& -\frac{1}{2 \mu\left[\left(1+\delta_{y}^{2}\right)+(n-1)\left(1+\rho \delta_{y}^{2}\right)\right]} \\
& \cdot E\left(c_{m_{2}} \mid c_{m_{1}}, c_{m_{2}}\right)-\frac{1}{2} E\left(q_{1 k}^{Y N *} \mid c_{m_{1}}, c_{m_{2}}\right) .
\end{aligned}
$$

With reference to Proposition 1 in [14], the unique equilibrium solutions for the two manufacturers are presented as follows:

$$
\begin{aligned}
q_{1 k}^{Y N *} & \frac{\left(a-w_{k}\right)}{3 \mu(1-\rho) \delta_{y}^{2}} \\
& -\frac{\left(1+\rho \delta_{y}^{2}\right) \sum_{k=1}^{n}\left(a-w_{k}\right)}{3 \mu(1-\rho) \delta_{y}^{2}\left[\left(1+\delta_{y}^{2}\right)+(n-1)\left(1+\rho \delta_{y}^{2}\right)\right]} \\
& +\frac{\eta-2}{3 \mu\left[\left(1+\delta_{y}^{2}\right)+(n-1)\left(1+\rho \delta_{y}^{2}\right)\right]} c_{m_{1},}^{Y N *} \\
= & \frac{\left(a-w_{k}\right)}{3 \mu(1-\rho) \delta_{y}^{2}} \quad\left(1+\rho \delta_{y}^{2}\right) \sum_{k=1}^{n}\left(a-w_{k}\right) \\
& -\frac{1}{3 \mu(1-\rho) \delta_{y}^{2}\left[\left(1+\delta_{y}^{2}\right)+(n-1)\left(1+\rho \delta_{y}^{2}\right)\right]} \\
& +\frac{2-\eta}{6 \mu\left[\left(1+\delta_{y}^{2}\right)+(n-1)\left(1+\rho \delta_{y}^{2}\right)\right]} c_{m_{1}} \\
& -\frac{\left.\left.1+\delta_{y}^{2}\right)+(n-1)\left(1+\rho \delta_{y}^{2}\right)\right]}{2 \mu\left[m_{2} .\right.}
\end{aligned}
$$

(2) The optimal decisions for the manufacturers at equilibrium are

$$
\begin{aligned}
& q_{1 k}^{Y N *}=\bar{q}_{1 k}+f_{1 k 1}^{Y N} c_{m_{1}}, \\
& q_{2 k}^{Y N *}=\bar{q}_{2 k}+f_{2 k 1}^{Y N} c_{m_{1}}+f_{2 k 2}^{Y N} c_{m_{2}},
\end{aligned}
$$

where

$$
\begin{aligned}
& f_{1 k 1}^{Y N}=\frac{\left[5(1-\rho) \delta_{y}^{2}+2(n-1)\left(1+\rho \delta_{y}^{2}\right)\right] \eta-\left[7(1-\rho) \delta_{y}^{2}+4(n-1)\left(1+\rho \delta_{y}^{2}\right)\right]}{6 \mu\left[\left(1+\delta_{y}^{2}\right)+(n-1)\left(1+\rho \delta_{y}^{2}\right)\right]\left[2(1-\rho) \delta_{y}^{2}+(n-1)\left(1+\rho \delta_{y}^{2}\right)\right]}, \\
& f_{2 k 1}^{Y N}=\frac{\left[5(1-\rho) \delta_{y}^{2}+2(n-1)\left(1+\rho \delta_{y}^{2}\right)\right]-\left[(1-\rho) \delta_{y}^{2}+(n-1)\left(1+\rho \delta_{y}^{2}\right)\right] \eta}{6 \mu\left[\left(1+\delta_{y}^{2}\right)+(n-1)\left(1+\rho \delta_{y}^{2}\right)\right]\left[2(1-\rho) \delta_{y}^{2}+(n-1)\left(1+\rho \delta_{y}^{2}\right)\right]}, \\
& f_{2 k 2}^{Y N}=-\frac{1}{2 \mu\left[\left(1+\delta_{y}^{2}\right)+(n-1)\left(1+\rho \delta_{y}^{2}\right)\right]} .
\end{aligned}
$$

Proposition 3 indicates that both the suppliers and manufacturer 1's optimal decisions only depend on $c_{m_{1}}$, while manufacturer 2's optimal decision depends on both $c_{m_{1}}$ and $c_{m_{2}}$. This is because manufacturer 2 can infer manufacturer 1 's cost information from $w_{k}^{Y N *}$, while manufacturer 1 cannot infer manufacturer 2's cost information from $w_{k}^{Y N *} \cdot \xi_{k 1}^{Y N}<$ $0, f_{i k i}^{Y N}<0$, and $f_{j k j}^{Y N}<0$, respectively, indicate that the suppliers, manufacturer 1 , and manufacturer 2 respond negatively to $c_{m_{1}}, c_{m_{1}}$, and $c_{m_{2}}$. Moreover, $f_{2 k 1}^{Y N}$ negatively or positively depends on the value of key parameters: $\delta_{y}, \rho$, and 
$t$. When $\left[5(1-\rho) \delta_{y}^{2}+2(n-1)\left(1+\rho \delta_{y}^{2}\right)\right]<\left[(1-\rho) \delta_{y}^{2}+\right.$ $\left.(n-1)\left(1+\rho \delta_{y}^{2}\right)\right] \eta$, manufacturer 2 responds negatively to $c_{m_{1}}$, while manufacturer 2 responds positively to $c_{m_{1}}$.

All information from Propositions $1-3$ is valuable to the suppliers and manufacturers in determining their wholesale prices and order quantities.

\section{Information Sharing Game}

We first calculate the suppliers and manufacturers' ex ante payoffs based on the equilibrium solutions for any pair of VCIS strategies and summarize the results in Table 1. Subsequently, we solve the cost-information sharing game.

Next, we analyze how manufacturers' VCIS affects each supplier by comparing SC partners' ex ante payoffs under strategy $(Y, N)$ with $(N, N)$ and comparing SC partners' ex ante payoffs under strategy $(Y, Y)$ with $(N, Y)$.

Proposition 4. The SC members' ex ante payoffs have the following properties.

(1) $\pi_{s_{k}}^{Y Y *}>\pi_{s_{k}}^{Y N *}>\pi_{s_{k}}^{N N *}$.

(2) (a) $\pi_{m_{1}}^{Y Y *}>\pi_{m_{1}}^{N Y *}$; (b) if $0<\eta<\eta_{1}, \pi_{m_{1}}^{Y N *}>\pi_{m_{1}}^{N N *}$; if $1 \geq \eta>\eta_{1}, \pi_{m_{1}}^{Y N *}<\pi_{m_{1}}^{N N *}$, where

$$
\eta_{1}=\frac{2(1-\rho) \delta_{y}^{2}+2(n-1)\left(1+\rho \delta_{y}^{2}\right)}{5(1-\rho) \delta_{y}^{2}+2(n-1)\left(1+\rho \delta_{y}^{2}\right)} .
$$

(3) (a) $\Pi_{m_{2}}^{Y Y *}>\pi_{m_{2}}^{N Y *}$; (b) if $\varphi\left(n, \rho, \delta_{y}\right)>\psi\left(n, \rho, \delta_{y}, \eta\right)$, $\pi_{m_{2}}^{Y N *}>\Pi_{m_{2}}^{N N *}$; if $\varphi\left(n, \rho, \delta_{y}\right)<\psi\left(n, \rho, \delta_{y}, \eta\right), \pi_{m_{2}}^{Y N *}<\Pi_{m_{2}}^{N N *}$, where

$$
\begin{aligned}
\varphi\left(n, \rho, \delta_{y}\right)= & \frac{n\left[5(1-\rho) \delta_{y}^{2}+2(n-1)\left(1+\rho \delta_{y}^{2}\right)\right]^{2} \sigma_{c}^{2}+9 n\left[2(1-\rho) \delta_{y}^{2}+(n-1)\left(1+\rho \delta_{y}^{2}\right)\right]^{2} \sigma_{c}^{2}}{36\left[\left(1+\delta_{y}^{2}\right)+(n-1)\left(1+\rho \delta_{y}^{2}\right)\right]\left[2(1-\rho) \delta_{y}^{2}+(n-1)\left(1+\rho \delta_{y}^{2}\right)\right]^{2}}, \\
\psi\left(n, \rho, \delta_{y}, \eta\right)= & \frac{n\left[7(1-\rho) \delta_{y}^{2}+4(n-1)\left(1+\rho \delta_{y}^{2}\right)\right]\left[5(1-\rho) \delta_{y}^{2}+2(n-1)\left(1+\rho \delta_{y}^{2}\right)\right] \eta \sigma_{c}^{2}}{18\left[\left(1+\delta_{y}^{2}\right)+(n-1)\left(1+\rho \delta_{y}^{2}\right)\right]\left[2(1-\rho) \delta_{y}^{2}+(n-1)\left(1+\rho \delta_{y}^{2}\right)\right]^{2}} \\
& -\frac{n\left[(1-\rho) \delta_{y}^{2}+(n-1)\left(1+\rho \delta_{y}^{2}\right)\right]\left[13(1-\rho) \delta_{y}^{2}+7(n-1)\left(1+\rho \delta_{y}^{2}\right)\right] \eta^{2} \sigma_{c}^{2}}{36\left[\left(1+\delta_{y}^{2}\right)+(n-1)\left(1+\rho \delta_{y}^{2}\right)\right]\left[2(1-\rho) \delta_{y}^{2}+(n-1)\left(1+\rho \delta_{y}^{2}\right)\right]^{2}} \\
& +\frac{n \sigma_{c}^{2}}{\left[\left(1+\delta_{y}^{2}\right)+(n-1)\left(1+\rho \delta_{y}^{2}\right)\right](2+\eta)^{2}} .
\end{aligned}
$$

Proof. See Appendix.

Proposition 4 Part (1) means that the suppliers will gain more ex ante payoffs from more manufacturers disclosing their private cost information.

Proposition 4 Part (2) shows that CCIS and NCIS are two possible equilibrium solutions for the two manufacturers. The manufacturers always agree to VCIS when the correlated coefficient of two manufacturers' cost uncertainty is less than a threshold (i.e., $0<\eta<\eta_{1}$ ).

Proposition 4 Part (3) states that a manufacturer does not always benefit from its competitor manufacturer's information sharing. If a manufacturer does not agree to VCIS, it benefits from the competitor manufacturer's VCIS only when $\varphi\left(n, \rho, \delta_{y}\right)>\psi\left(n, \rho, \delta_{y}, \eta\right)$.

Proposition 5. Complete cost-information sharing Paretodominates no cost-information sharing.

Proof. See Appendix.

Proposition 5 suggests that the entire SC's ex ante payoff with CCIS is larger than NCIS.

\section{Numerical Analysis}

In this section, we examine the impact of key parameters on the value of information sharing. As the two manufacturers are symmetric, we only focus on the value of information sharing by manufacturer 1 . Let $V_{s_{k}}^{N}=\Pi_{s_{k}}^{Y N *}-\Pi_{s_{k}}^{N N *}, V_{m_{2}}^{N}=$ $\Pi_{m_{2}}^{Y N *}-\Pi_{m_{2}}^{N N *}$, and $V_{m_{1}}^{N}=\Pi_{m_{1}}^{Y N *}-\Pi_{m_{1}}^{N N *}$, respectively, represent the effect of manufacturer 1's information sharing to each supplier, manufacturer 2, and manufacturer 1. Similarly, let $V_{s_{k}}^{Y}=\Pi_{s_{k}}^{Y Y *}-\Pi_{s_{k}}^{N Y *}, V_{m_{2}}^{Y}=\Pi_{m_{2}}^{Y Y *}-\Pi_{m_{2}}^{N Y *}$, and $V_{m_{1}}^{Y}=$ $\Pi_{m_{1}}^{Y Y{ }^{*}}-\Pi_{m_{1}}^{N{ }^{*}}$, respectively, denote the effect of manufacturer 1 information sharing on SC partners' ex ante payoffs.

We assume the following: $\sigma_{\theta}^{2}=2, \rho=0.5, \delta_{y}=0.5, \eta=$ 0.7 , and $n=2$. The effects of $\rho, \delta_{y}, \eta$, and $n$ on $V_{s_{k}}^{N}, V_{m_{2}}^{N}, V_{m_{1}}^{N}$, $V_{s_{k}}^{Y}, V_{m_{2}}^{Y}$, and $V_{m_{1}}^{Y}$ are provided in Figures 1-4, respectively.

Figures 1(a) and 1(b) show that as $\rho$ increases, $V_{s_{k}}^{N}$ and $V_{s_{k}}^{Y}$ decreases. It means that, whether manufacture 2 shares information or not, the higher $\rho$ is, the less each supplier benefits from information sharing by manufacturer 1 .

Figures $1(\mathrm{c})$ and $1(\mathrm{~d})$ show that as $\rho$ increases, $V_{m_{2}}^{N}$ increases, while $V_{m_{2}}^{Y}$ decreases. This shows that if manufacture 
TABLE 1: The manufacturers' and suppliers' ex ante payoffs.

\begin{tabular}{|c|c|c|}
\hline Subgame & The suppliers & The manufacturers \\
\hline$(Y, Y)$ & $\Pi_{s_{k}}^{Y Y *}=\bar{\Pi}_{s_{k}}+\frac{W R(\eta+1) \sigma_{c}^{2}}{3 B C^{2}}$ & $\Pi_{m_{i}}^{Y Y *}=\bar{\Pi}_{m_{i}}+\frac{n\left(U^{2}+T^{2}\right) \sigma_{c}^{2}}{36 B C^{2}}-\frac{n U T \eta \sigma_{c}^{2}}{18 B C^{2}}$ \\
\hline$(N, N)$ & $\Pi_{s_{k}}^{N N *}=\bar{\Pi}_{s_{k}}$ & $\Pi_{m_{i}}^{N N *}=\bar{\Pi}_{m_{i}}+\frac{n \sigma_{c}^{2}}{B(2+\eta)^{2}}$ \\
\hline$(Y, N)$ & $\Pi_{s_{k}}^{Y N *}=\bar{\Pi}_{s_{k}}+\frac{W R(\eta+1)^{2} \sigma_{c}^{2}}{6 B C^{2}}$ & $\begin{array}{c}\Pi_{m_{1}}^{Y N *}=\bar{\Pi}_{m_{i}}+\frac{n(\eta T-U)^{2}}{36 B C^{2}} \sigma_{c}^{2} \\
\Pi_{m_{2}}^{Y N *}=\bar{\Pi}_{m_{i}}+\frac{n(T-\eta R)^{2} \sigma_{c}^{2}}{36 B C^{2}}+\frac{n \sigma_{c}^{2}}{4 B}-\frac{n \eta(T-\eta R) \sigma_{c}^{2}}{6 B C}\end{array}$ \\
\hline$(N, Y)$ & $\Pi_{s_{k}}^{N Y *}=\bar{\Pi}_{s_{k}}+\frac{W R(\eta+1)^{2} \sigma_{c}^{2}}{6 B C^{2}}$ & $\begin{array}{c}\Pi_{m_{1}}^{N Y *}=\bar{\Pi}_{m_{1}}+\frac{n(T-\eta R)^{2} \sigma_{c}^{2}}{36 B C^{2}}+\frac{n \sigma_{c}^{2}}{4 B}-\frac{n \eta(T-\eta R) \sigma_{c}^{2}}{6 B C} \\
\Pi_{m_{2}}^{N Y *}=\bar{\Pi}_{m_{2}}+\frac{n(\eta T-U)^{2} \sigma_{c}^{2}}{36 B C^{2}}\end{array}$ \\
\hline
\end{tabular}

Notes. $\bar{\Pi}_{m_{i}}=\left(n R^{2} / 9 B C^{2}\right)(a-c)^{2}$ and $\bar{\Pi}_{s_{k}}=\left(2(1-\rho) \delta_{y}^{2} R / 3 B C^{2}\right)(a-c)^{2}, i=1,2, k=1,2, \ldots, n ; B=\left(1+\delta_{y}^{2}\right)+(n-1)\left(1+\rho \delta_{y}^{2}\right)$ and $C=2(1-\rho) \delta_{y}^{2}+(n-$ $1)\left(1+\rho \delta_{y}^{2}\right) ; R=(1-\rho) \delta_{y}^{2}+(n-1)\left(1+\rho \delta_{y}^{2}\right)$ and $T=5(1-\rho) \delta_{y}^{2}+2(n-1)\left(1+\rho \delta_{y}^{2}\right) ; U=7(1-\rho) \delta_{y}^{2}+4(n-1)\left(1+\rho \delta_{y}^{2}\right)$ and $W=(1-\rho) \delta_{y}^{2}$.

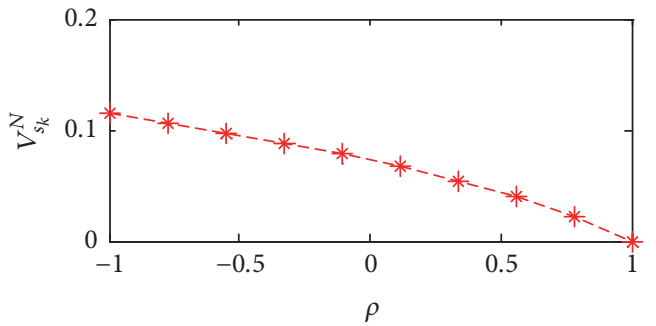

(a)

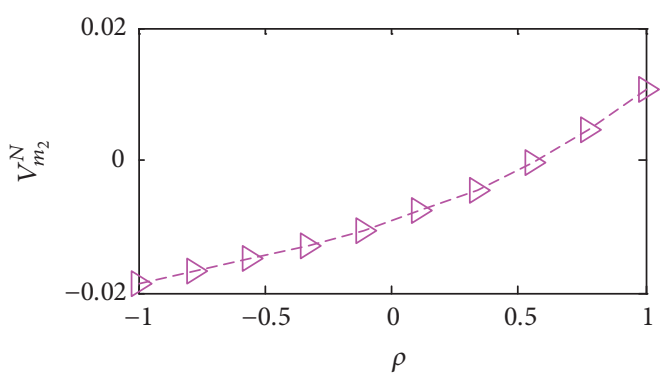

(c)

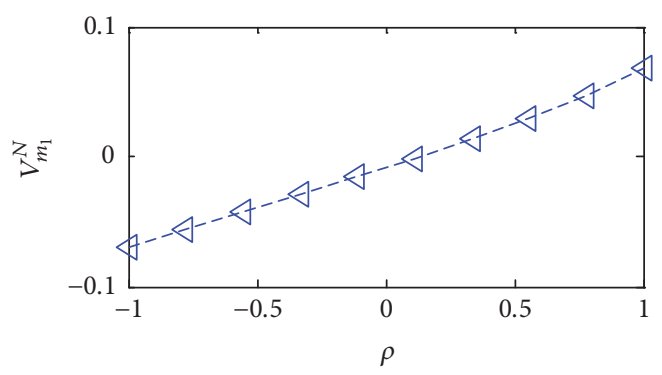

(e)

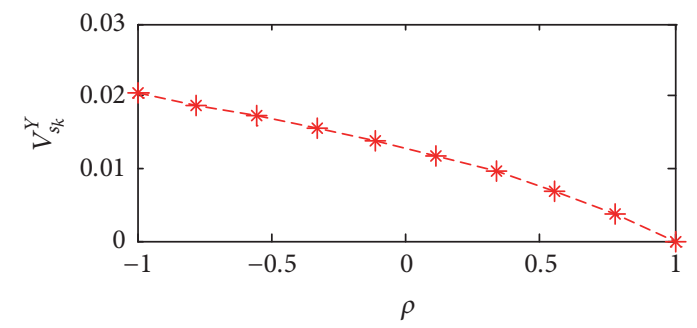

(b)

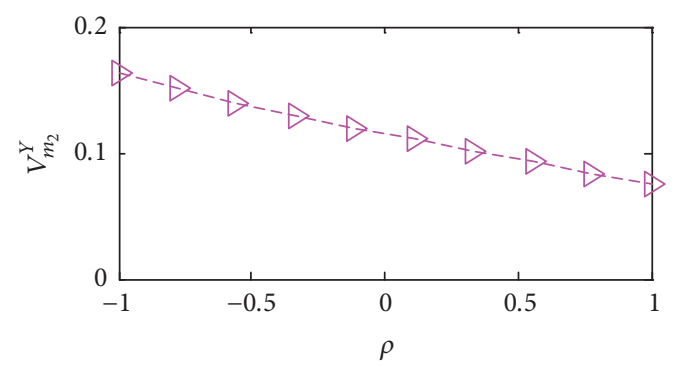

(d)

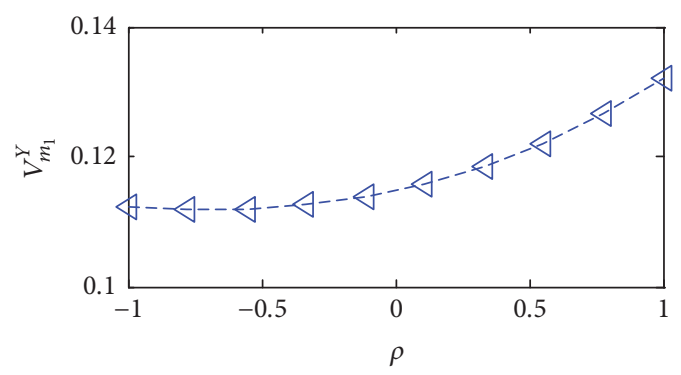

(f)

FIGURE 1: The effect of $\rho$ on $V_{s_{k}}^{N}, V_{m_{2}}^{N}, V_{m_{1}}^{N}, V_{s_{k}}^{Y}, V_{m_{2}}^{Y}$, and $V_{m_{1}}^{Y}$. 


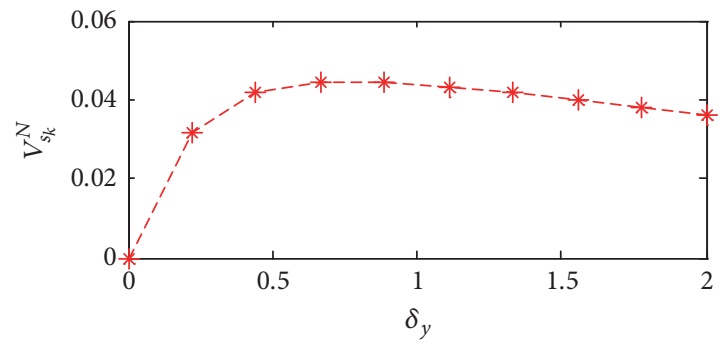

(a)

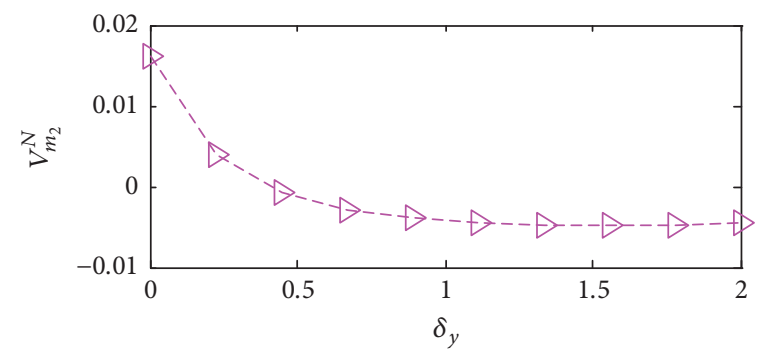

(c)

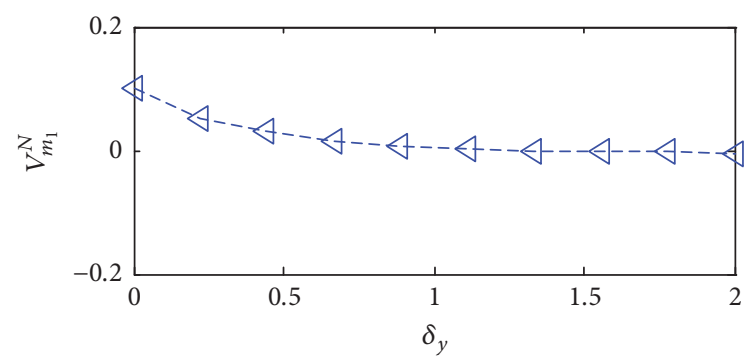

(e)

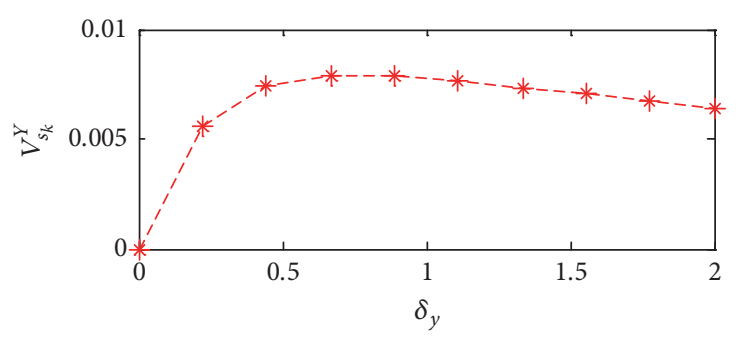

(b)

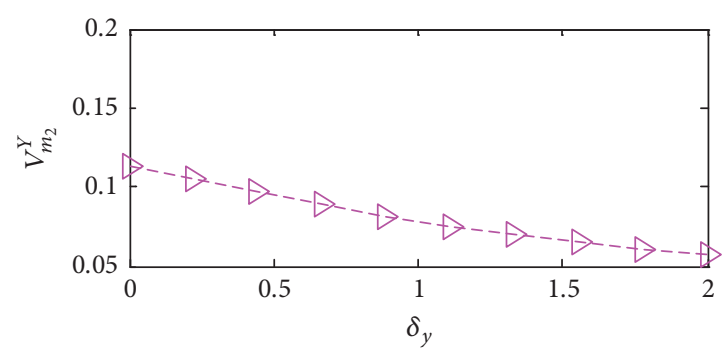

(d)

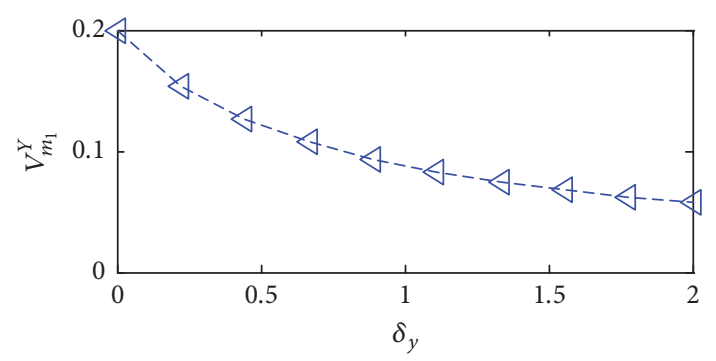

(f)

FIGURE 2: The impact of $\delta_{y}$ on $V_{s_{k}}^{N}, V_{m_{2}}^{N}, V_{m_{1}}^{N}, V_{s_{k}}^{Y}, V_{m_{2}}^{Y}$, and $V_{m_{1}}^{Y}$.

2 agrees to VCIS, high $\rho$ increases manufacturer 2's benefits from manufacturer 1's VCIS. If it does not agree to VCIS, high $\rho$ reduces manufacturer 2's benefits from manufacturer 1's VCIS.

Figures 1(e) and 1(f) show that both $V_{m_{1}}^{N}$ and $V_{m_{1}}^{Y}$ increase with $\rho$. These mean that high $\rho$ increases the value of information sharing by manufacturer 1 to itself.

Figures 2(a) and 2(b) show that as $\delta_{y}$ increases, both of $V_{s_{k}}^{N}$ and $V_{s_{k}}^{Y}$ first increase and then decrease. This indicates that the impacts of $\delta_{y}$ on $V_{s_{k}}^{N}$ and $V_{s_{k}}^{Y}$ are in the same direction.

Figures 2(c)-2(f) show that as $\delta_{y}$ increases, $V_{m_{2}}^{N}, V_{m_{2}}^{Y}$, $V_{m_{1}}^{N}$, and $V_{m_{1}}^{Y}$ decrease. This means that high $\delta_{y}$ will decrease the value of information sharing by manufacturer 1 to both manufacturer 1 and manufacturer 2 .

Figures 3(a) and 3(b) show that $V_{s_{k}}^{N}$ increases with $\eta$, while $V_{s_{k}}^{Y}$ decreases with $\eta$. This means that given the fact that manufacture 2 decides not to disclose its cost information to its suppliers, high $\eta$ promotes manufacturer 2's benefit when manufacturer 1 shares its cost information. If manufacture 2 decides to disclose its private cost information to its suppliers, high $\rho$ decreases manufacturer 2's benefit when manufacturer 1 shares its cost information.

Figures 3(c)-3(f) show that as $\eta$ increases, $V_{m_{2}}^{N}, V_{m_{2}}^{Y}, V_{m_{1}}^{N}$, and $V_{m_{1}}^{Y}$ decrease. It means that no matter whether manufacturer 2 decides to disclose cost information to suppliers or not, the value of information sharing by manufacturer 1 to both manufacturer 1 and manufacturer 2 decreases with $\eta$.

Figures $4(\mathrm{a})$ and $4(\mathrm{~b})$ show that $V_{s_{k}}^{N}$ and $V_{s_{k}}^{Y}$ decrease with $n$. Figures $4(\mathrm{e})$ and $4(\mathrm{f})$ show that $V_{m_{1}}^{N}$ and $V_{m_{1}}^{Y}$ increase with $n$. It means that as the number of suppliers increases, each supplier benefits less from information sharing by manufacturer 1 , while manufacturer 1 benefits more from information sharing by itself.

Figures $4(\mathrm{c})$ and $4(\mathrm{~d})$ show that as $n$ increases, $V_{m_{2}}^{N}$ increases while $V_{m_{2}}^{Y}$ decreases. It means that as the number of suppliers increases, the value of information sharing by manufacturer 1 is determined by manufacturer 2's information sharing strategy. 


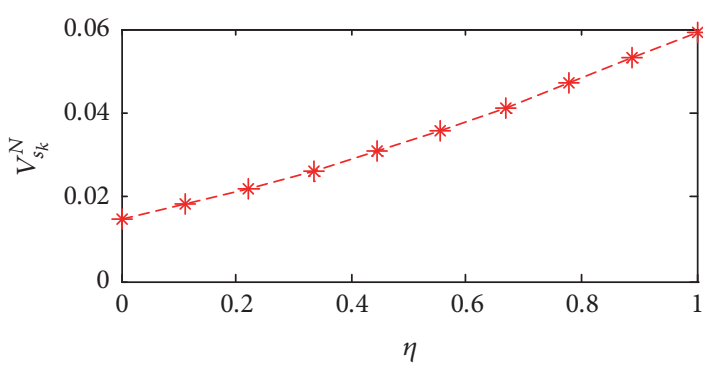

(a)

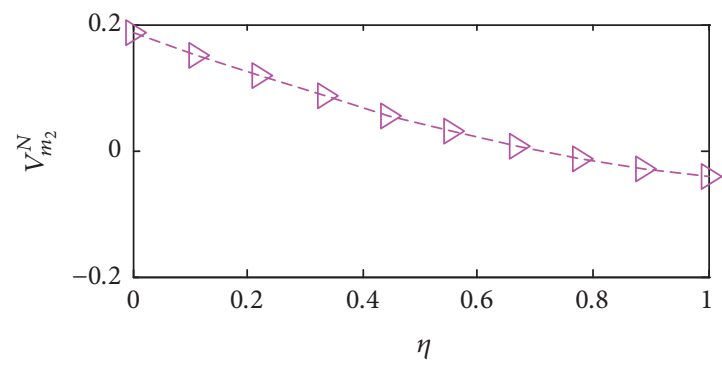

(c)

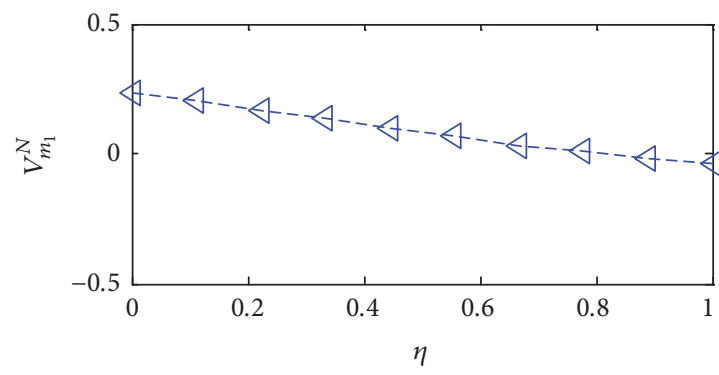

(e)

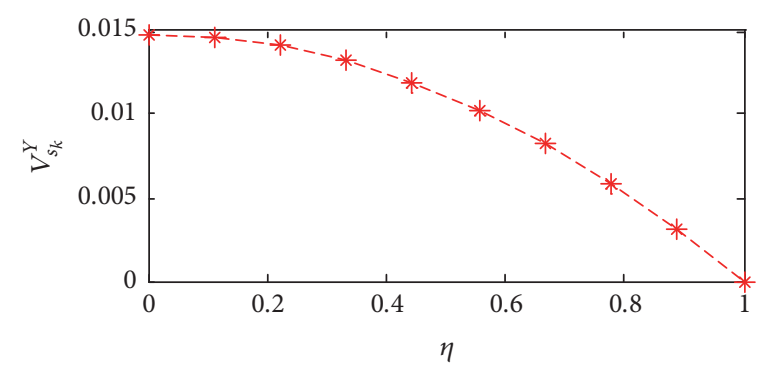

(b)

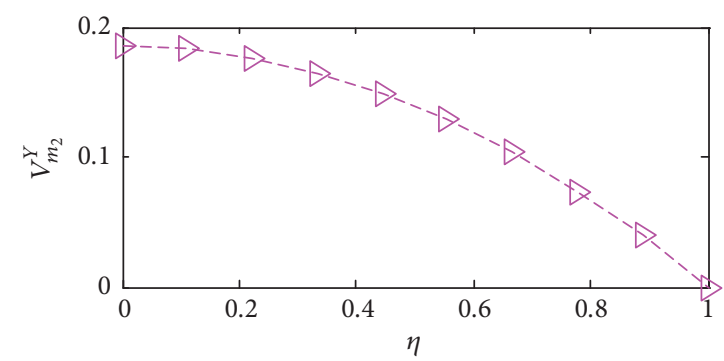

(d)

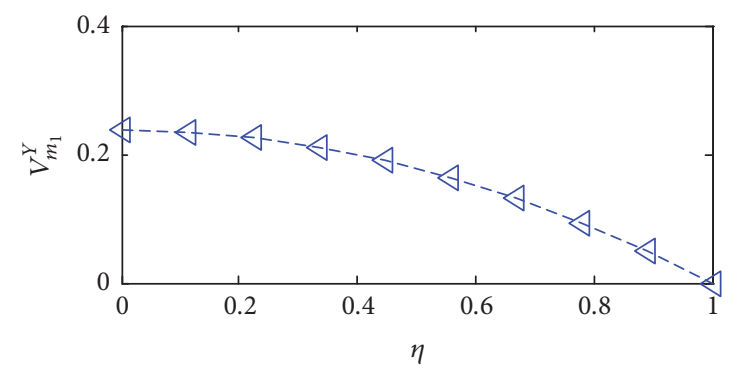

(f)

FIGURE 3: The impact of $\eta$ on $V_{s_{k}}^{N}, V_{m_{2}}^{N}, V_{m_{1}}^{N}, V_{s_{k}}^{Y}, V_{m_{2}}^{Y}$, and $V_{m_{1}}^{Y}$.

Figures 1-4 show that $V_{s_{k}}^{N}, V_{s_{k}}^{Y}, V_{m_{2}}^{Y}$, and $V_{m_{1}}^{Y}$ are positive. Whether $V_{m_{2}}^{N}$ and $V_{m_{1}}^{N}$ are positive or negative is determined by the value of key parameters. $V_{m_{1}}^{Y}>0$ indicates that if manufacturer 2 chooses to disclose its cost information to suppliers, manufacturer 1 will also reveal its cost information to suppliers. $V_{m_{1}}^{N}<0$ suggests that given the fact that manufacturer 2 does not share information with suppliers, neither will manufacturer 1 with its own suppliers.

Moreover, we found that if manufacturer 1 shares its cost information, higher $\rho$ means that manufacturer 1 would be more willing to share information (see Figure 1(f)), promoting complete information sharing. Similarly, higher $n$ also promotes complete information sharing (see Figure 4(f)). However, higher $\delta_{y}$ and $\eta$ undermine complete information sharing (see Figures 2(f) and 3(f)).

\section{Conclusions}

Information sharing is a hot topic in the literature of SC management. This study examines VCIS in a simplified SC which consists of two manufacturers with private cost information and $n$ suppliers with yield uncertainty.
This work contributes to the area of research on incentive for VCIS. We analyze VCIS by considering the number of suppliers, the correlated coefficient of manufacturers' cost uncertainty, and the level of yield uncertainty and the correlated coefficient of the supply processes. We found that there exists only two equilibrium information sharing strategies: complete cost-information sharing and no costinformation sharing. The manufactures always agree to VCIS when the correlated coefficient of two manufacturers' cost uncertainty is less than a threshold. In addition, complete cost-information sharing will increase each supplier, each manufacturer, and the entire SC's ex ante payoffs when two manufacturers' cost uncertainty is less correlated. It suggests that the manufactures decide to perform VCIS.

This presented study can be further extended along the following three directions. First, other types of contract (e.g., two-part pricing contract) can be considered. Second, multiple manufacturers could be introduced to examine how the number of manufacturers affects the manufacturers' willingness to share information. Finally, our model could be expanded to include other types of competition such as newsvendor competition models. 


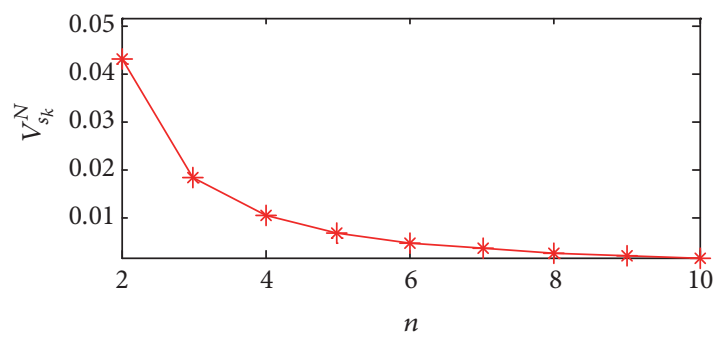

(a)

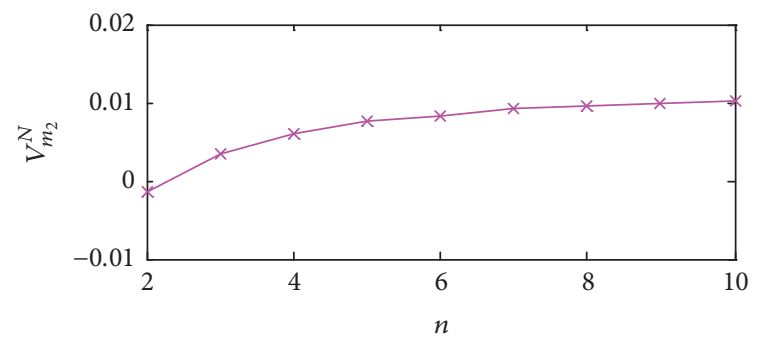

(c)

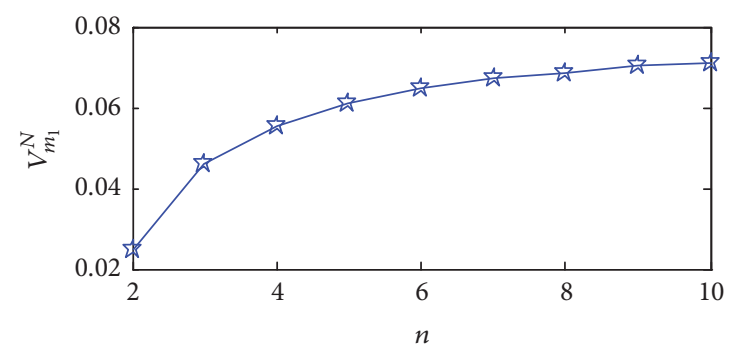

(e)

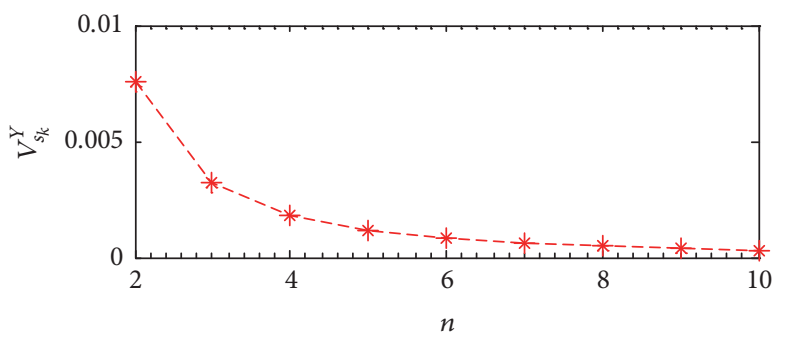

(b)

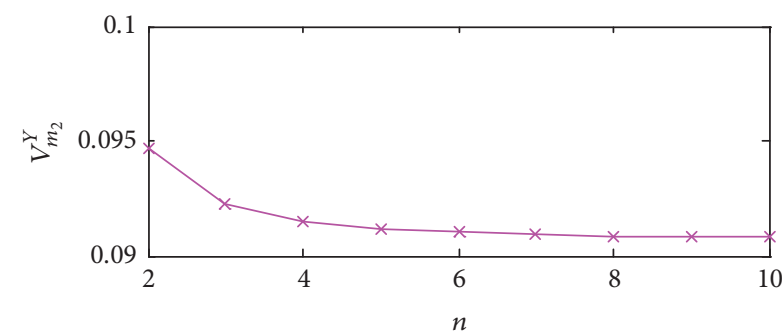

(d)

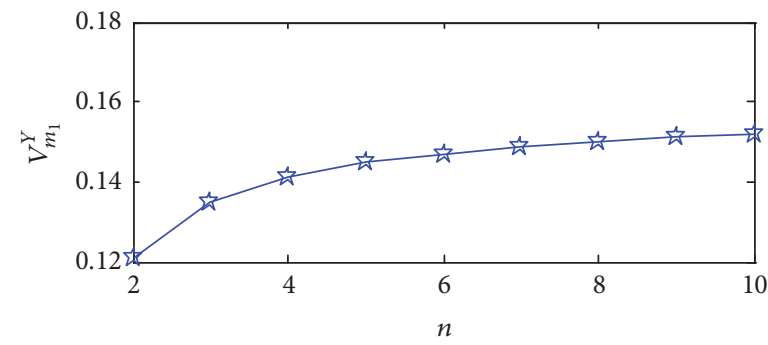

(f)

FIGURE 4: The impact of $n$ on $V_{s_{k}}^{N}, V_{m_{2}}^{N}, V_{m_{1}}^{N}, V_{s_{k}}^{Y}, V_{m_{2}}^{Y}$, and $V_{m_{1}}^{Y}$.

\section{Appendix}

Proof of Proposition 4. From Table 1, we have

$$
\begin{aligned}
& \Pi_{s_{k}}^{Y N *}-\Pi_{s_{k}}^{N N *}=\frac{(1-\rho) \delta_{y}^{2}\left[(1-\rho) \delta_{y}^{2}+(n-1)\left(1+\rho \delta_{y}^{2}\right)\right](\eta+1)^{2} \sigma_{c}^{2}}{6\left[\left(1+\delta_{y}^{2}\right)+(n-1)\left(1+\rho \delta_{y}^{2}\right)\right]\left[2(1-\rho) \delta_{y}^{2}+(n-1)\left(1+\rho \delta_{y}^{2}\right)\right]^{2}}>0 \\
& \Pi_{s_{k}}^{Y Y *}-\Pi_{s_{k}}^{Y N *}=\frac{(1-\rho) \delta_{y}^{2}\left[(1-\rho) \delta_{y}^{2}+(n-1)\left(1+\rho \delta_{y}^{2}\right)\right]\left(1-\eta^{2}\right) \sigma_{c}^{2}}{6\left[\left(1+\delta_{y}^{2}\right)+(n-1)\left(1+\rho \delta_{y}^{2}\right)\right]\left[2(1-\rho) \delta_{y}^{2}+(n-1)\left(1+\rho \delta_{y}^{2}\right)\right]^{2}}>0 \\
& \Pi_{m_{2}}^{Y N *}-\Pi_{m_{2}}^{N N *} \\
& =\frac{n\left[5(1-\rho) \delta_{y}^{2}+2(n-1)\left(1+\rho \delta_{y}^{2}\right)\right]^{2} \sigma_{c}^{2}+9 n\left[2(1-\rho) \delta_{y}^{2}+(n-1)\left(1+\rho \delta_{y}^{2}\right)\right]^{2} \sigma_{c}^{2}}{36\left[\left(1+\delta_{y}^{2}\right)+(n-1)\left(1+\rho \delta_{y}^{2}\right)\right]\left[2(1-\rho) \delta_{y}^{2}+(n-1)\left(1+\rho \delta_{y}^{2}\right)\right]^{2}} \\
& \quad+\frac{n\left[(1-\rho) \delta_{y}^{2}+(n-1)\left(1+\rho \delta_{y}^{2}\right)\right]\left[13(1-\rho) \delta_{y}^{2}+7(n-1)\left(1+\rho \delta_{y}^{2}\right)\right] \eta^{2} \sigma_{c}^{2}}{36\left[\left(1+\delta_{y}^{2}\right)+(n-1)\left(1+\rho \delta_{y}^{2}\right)\right]\left[2(1-\rho) \delta_{y}^{2}+(n-1)\left(1+\rho \delta_{y}^{2}\right)\right]^{2}} \\
& \quad-\frac{n\left[7(1-\rho) \delta_{y}^{2}+4(n-1)\left(1+\rho \delta_{y}^{2}\right)\right]\left[5(1-\rho) \delta_{y}^{2}+2(n-1)\left(1+\rho \delta_{y}^{2}\right)\right] \eta \sigma_{c}^{2}}{18\left[\left(1+\delta_{y}^{2}\right)+(n-1)\left(1+\rho \delta_{y}^{2}\right)\right]\left[2(1-\rho) \delta_{y}^{2}+(n-1)\left(1+\rho \delta_{y}^{2}\right)\right]^{2}}-\frac{n \sigma_{c}^{2}}{\left[\left(1+\delta_{y}^{2}\right)+(n-1)\left(1+\rho \delta_{y}^{2}\right)\right](2+\eta)^{2}}
\end{aligned}
$$




$$
\begin{aligned}
& \Pi_{m_{2}}^{Y Y *}-\pi_{m_{2}}^{N Y *}=\frac{n\left[5(1-\rho) \delta_{y}^{2}+2(n-1)\left(1+\rho \delta_{y}^{2}\right)\right]^{2}\left(1-\eta^{2}\right) \sigma_{c}^{2}}{36\left[\left(1+\delta_{y}^{2}\right)+(n-1)\left(1+\rho \delta_{y}^{2}\right)\right]\left[2(1-\rho) \delta_{y}^{2}+(n-1)\left(1+\rho \delta_{y}^{2}\right)\right]^{2}}>0, \\
& \pi_{m_{1}}^{Y N *}-\pi_{m_{1}}^{N N *} \\
& =\frac{-n\left\{(1-\rho) \delta_{y}^{2}\left(26-3 \eta-5 \eta^{2}\right)+2(n-1)\left(1+\rho \delta_{y}^{2}\right)\left(7-\eta^{2}\right)\right\} \cdot\left\{3(1-\rho) \delta_{y}^{2} \eta-2\left[(1-\rho) \delta_{y}^{2}+(n-1)\left(1+\rho \delta_{y}^{2}\right)\right](1-\eta)\right\}(1+\eta) \sigma_{c}^{2}}{36\left[\left(1+\delta_{y}^{2}\right)+(n-1)\left(1+\rho \delta_{y}^{2}\right)\right]\left[2(1-\rho) \delta_{y}^{2}+(n-1)\left(1+\rho \delta_{y}^{2}\right)\right]^{2}(2+\eta)^{2}}, \\
& \pi_{m_{1}}^{Y Y *}-\pi_{m_{1}}^{N Y *}=\frac{n\left[(1-\rho) \delta_{y}^{2}+(n-1)\left(1+\rho \delta_{y}^{2}\right)\right]\left[13(1-\rho) \delta_{y}^{2}+7(n-1)\left(1+\rho \delta_{y}^{2}\right)\right]\left(1-\eta^{2}\right) \sigma_{c}^{2}}{36\left[\left(1+\delta_{y}^{2}\right)+(n-1)\left(1+\rho \delta_{y}^{2}\right)\right]\left[2(1-\rho) \delta_{y}^{2}+(n-1)\left(1+\rho \delta_{y}^{2}\right)\right]^{2}}>0 .
\end{aligned}
$$

In (A.3), let $\Pi_{m_{2}}^{Y N *}-\Pi_{m_{2}}^{N N *}=0$, and we obtain $\varphi\left(n, \rho, \delta_{y}\right)=$ $\psi\left(n, \rho, \delta_{y}, \eta\right)$, where

$$
\begin{aligned}
\varphi\left(n, \rho, \delta_{y}\right)= & \frac{n\left[5(1-\rho) \delta_{y}^{2}+2(n-1)\left(1+\rho \delta_{y}^{2}\right)\right]^{2} \sigma_{c}^{2}+9 n\left[2(1-\rho) \delta_{y}^{2}+(n-1)\left(1+\rho \delta_{y}^{2}\right)\right]^{2} \sigma_{c}^{2}}{36\left[\left(1+\delta_{y}^{2}\right)+(n-1)\left(1+\rho \delta_{y}^{2}\right)\right]\left[2(1-\rho) \delta_{y}^{2}+(n-1)\left(1+\rho \delta_{y}^{2}\right)\right]^{2}}, \\
\psi\left(n, \rho, \delta_{y}, \eta\right)= & \frac{n\left[7(1-\rho) \delta_{y}^{2}+4(n-1)\left(1+\rho \delta_{y}^{2}\right)\right]\left[5(1-\rho) \delta_{y}^{2}+2(n-1)\left(1+\rho \delta_{y}^{2}\right)\right] \eta \sigma_{c}^{2}}{18\left[\left(1+\delta_{y}^{2}\right)+(n-1)\left(1+\rho \delta_{y}^{2}\right)\right]\left[2(1-\rho) \delta_{y}^{2}+(n-1)\left(1+\rho \delta_{y}^{2}\right)\right]^{2}} \\
& -\frac{n\left[(1-\rho) \delta_{y}^{2}+(n-1)\left(1+\rho \delta_{y}^{2}\right)\right]\left[13(1-\rho) \delta_{y}^{2}+7(n-1)\left(1+\rho \delta_{y}^{2}\right)\right] \eta^{2} \sigma_{c}^{2}}{36\left[\left(1+\delta_{y}^{2}\right)+(n-1)\left(1+\rho \delta_{y}^{2}\right)\right]\left[2(1-\rho) \delta_{y}^{2}+(n-1)\left(1+\rho \delta_{y}^{2}\right)\right]^{2}} \\
& +\frac{n \sigma_{c}^{2}}{\left[\left(1+\delta_{y}^{2}\right)+(n-1)\left(1+\rho \delta_{y}^{2}\right)\right](2+\eta)^{2}} .
\end{aligned}
$$
where

In (A.5), let $\pi_{m_{1}}^{Y N *}-\pi_{m_{1}}^{N N *}=0$, and we obtain $\eta=\eta_{1}$,

$$
\eta_{1}=\frac{2(1-\rho) \delta_{y}^{2}+2(n-1)\left(1+\rho \delta_{y}^{2}\right)}{5(1-\rho) \delta_{y}^{2}+2(n-1)\left(1+\rho \delta_{y}^{2}\right)} .
$$

Thus, we have

(1) $\pi_{s_{k}}^{Y Y *}>\pi_{s_{k}}^{Y N *}>\pi_{s_{k}}^{N N *}$.

(2) (a) $\pi_{m_{1}}^{Y Y *}>\pi_{m_{1}}^{N Y *}$; (b) if $\eta<\eta_{1}, \pi_{m_{1}}^{Y N *}>\pi_{m_{1}}^{N N *}$; if $\eta>\eta_{1}$, $\pi_{m_{1}}^{Y N *}<\pi_{m_{1}}^{N N *}$.

(3) (a) $\Pi_{m_{2}}^{Y Y *}>\pi_{m_{2}}^{N Y *}$; (b) if $\varphi\left(n, \rho, \delta_{y}\right)>\psi\left(n, \rho, \delta_{y}, \eta\right)$, $\pi_{m_{2}}^{Y N *}>\Pi_{m_{2}}^{N N *}$; if $\varphi\left(n, \rho, \delta_{y}\right)<\psi\left(n, \rho, \delta_{y}, \eta\right), \pi_{m_{2}}^{Y N *}<\Pi_{m_{2}}^{N N *}$. The proof of Proposition 4 is finished.

Proof of Proposition 5. From Table 1, we obtain

$$
\begin{gathered}
\Pi^{Y Y *}-\Pi^{N N *}=\left(\pi_{m_{1}}^{Y Y *}+\pi_{m_{2}}^{Y Y *}+n \pi_{s_{k}}^{Y Y *}\right)-\left(\Pi_{m_{1}}^{N N *}\right. \\
\left.+\Pi_{m_{2}}^{N N *}+n \Pi_{s_{k}}^{N N *}\right)=\left(n \left\{2 \left[4(1-\rho)^{2} \delta_{y}^{4}\right.\right.\right. \\
\left.+(n-1)^{2}\left(1+\rho \delta_{y}^{2}\right)^{2}\right]\left[30 \eta^{2}+37 \eta(1-\eta)\right.
\end{gathered}
$$

$$
\left.+11(1-\eta)^{2}\right](1-\eta)+(n-1)(1-\rho) \delta_{y}^{2}(1
$$$$
\begin{aligned}
& \left.+\rho \delta_{y}^{2}\right)\left[18 \eta^{3}+273 \eta^{2}(1-\eta)+316 \eta(1-\eta)^{2}\right. \\
& \left.\left.\left.+92(1-\eta)^{3}\right]\right\}\left(1 / \eta^{2}\right) \sigma_{c}^{2}\right)\left(9 \left[\left(1+\delta_{y}^{2}\right)+(n-1)\right.\right.
\end{aligned}
$$$$
\left.\cdot\left(1+\rho \delta_{y}^{2}\right)\right]\left[2(1-\rho) \delta_{y}^{2}+(n-1)\left(1+\rho \delta_{y}^{2}\right)\right]^{2}
$$$$
\left.\cdot(3+2 t)^{2}\right)^{-1}>0
$$

The proof of Proposition 5 is finished.

\section{Notation for Variables}

$w_{k}, q_{i k}: \quad$ Wholesale price and order quantity (decision variable)

$\pi_{s_{k}}, \pi_{r_{i}}: \quad$ Supplier $k$ 's and manufacturer i's profit

$\bar{w}_{k}, \bar{q}_{i k}: \quad$ The equilibrium wholesale price and order quantity under no cost uncertainty

$w_{k}^{Z_{1} Z_{2} *}, q_{i k}^{Z_{1} Z_{2} *}:$ The equilibrium wholesale price and order quantity under subgame $\left(Z_{1}, Z_{2}\right)$ 
$\begin{array}{ll}\xi_{k i}^{Z_{1} Z_{2}}, f_{i k i}^{Z_{1} Z_{2}}: & \begin{array}{l}\text { Response coefficients for supplier } k \text { and } \\ \text { manufacturer } i \text { to } c_{m_{i}} \text { under subgame } \\ \left(Z_{1}, Z_{2}\right)\end{array} \\ \Pi_{s_{k}}^{Z_{1} Z_{2} *}, \Pi_{r_{i}}^{Z_{1} Z_{2} *}: \begin{array}{l}\text { The optimal ex ante payoffs for supplier } k \\ \text { and manufacturer } i \text { under subgame }\end{array} \\ \bar{\Pi}_{s_{k}}, \bar{\Pi}_{r_{i}}: & \begin{array}{l}\left(Z_{1}, Z_{2}\right) \\ \text { The optimal profit for supplier } k \text { and } \\ \text { manufacturer } i \text { under no cost uncertainty. }\end{array}\end{array}$

Parameters

$\begin{array}{ll}a: & \text { Demand intercept } \\ c_{m_{1}}: & \text { Cost uncertainty for manufacturer } i \\ \sigma_{c}^{2}: & \text { Variance of } c_{m_{i}} \\ y_{k}: & \text { Yield uncertainty } \\ \mu, \sigma_{y}^{2}: & \text { Mean and variance of } y_{k} \\ \delta_{y}: & \text { The level of yield uncertainty } \delta_{y}=\sigma_{y}^{2} / \mu^{2} \\ \rho: & \text { Correlated coefficient of suppliers' supply } \\ \eta: & \text { processes } \\ & \text { Correlated coefficient of manufacturers' } \\ c: & \text { Cost uncertainty }\end{array}$

Indices

Subscript

$s_{k}:$ It captures supplier $k$

$m_{i}$ : It captures manufacturer $i$

\section{Superscript}

$Y$ : It indicates that the manufacturer agrees to VCIS

$N$ : It indicates that the manufacturer does not agree to VCIS.

\section{Conflicts of Interest}

The authors declare that they have no conflicts of interest.

\section{Acknowledgments}

This work is supported by the National Natural Science Foundation of China [Grant nos. 71531004, 71390335] and the Scientific Innovation Research of College Graduates in Jiangsu Province [Grant no. CXLX13-097].

\section{References}

[1] H. L. Lee and S. Whang, "Information sharing in a supply chain," International Journal of Manufacturing Technology and Management, vol. 1, no. 1, pp. 79-93, 2000.

[2] L. Li and H. T. Zhang, "Confidentiality and information sharing in supply chain coordination," Management Science, vol. 54, no. 8, pp. 1467-1481, 2008.

[3] K. Zhu, "Information transparency of business-to-business electronic markets: a game-theoretic analysis," Management Science, vol. 50, no. 5, pp. 670-685, 2004.

[4] J. Recha, J. Kinyangi, and H. Omondi, "Climate related risks and opportunities for agricultural adaptation in semi-arid eastern Kenya," CCAFS East Africa Program Project Report, CGIAR Research Program on Climate Change, Agriculture and Food Security (CCAFS), Copenhagen, Denmark, 2013, http://hdl.handle.net/10568/24695.

[5] E. Y. Nyamah, Y. Jiang, Y. Feng, and E. Enchill, "Agri-food supply chain performance: an empirical impact of risk," Management Decision, vol. 55, no. 5, pp. 872-891, 2017.

[6] C. A. MacKenzie and A. Apte, "Modeling disruption in a fresh produce supply chain," The International Journal of Logistics Management, vol. 28, no. 2, pp. 656-679, 2017.

[7] M. de Keizer, R. Akkerman, M. Grunow, J. M. Bloemhof, R. Haijema, and J. G. van der Vorst, "Logistics network design for perishable products with heterogeneous quality decay," European Journal of Operational Research, vol. 262, no. 2, pp. 535-549, 2017.

[8] S. Jin, Y. Zhang, and Y. Xu, "Amount of information and the willingness of consumers to pay for food traceability in China," Food Control, vol. 77, pp. 163-170, 2017.

[9] F. Chen, "Information sharing and supply chain coordination," in Handbooks in Operations Research and Management Science, vol. 11, pp. 341-421, 2003.

[10] R. N. Clarke, "Collusion and the incentives for information sharing," The Bell Journal of Economics, vol. 14, no. 2, pp. 383394, 1983.

[11] E. Galor, "Information sharing in oligopoly," Econometrica, vol. 53, no. 2, pp. 329-343, 1985.

[12] A. J. Kirby, "Trade associations as information exchange mechanisms," The RAND Journal of Economics, vol. 19, no. 1, pp. 138$146,1988$.

[13] X. Vives, "Duopoly information equilibrium: Cournot and Bertrand," Journal of Economic Theory, vol. 34, no. 1, pp. 71-94, 1984.

[14] L. Li, "Cournot oligopoly with information sharing," The RAND Journal of Economics, vol. 16, no. 4, pp. 521-536, 1985.

[15] Z. Z. Zhou and K. X. Zhu, "The effects of information transparency on suppliers, manufacturers, and consumers in online markets," Marketing Science, vol. 29, no. 6, pp. 1125-1137, 2010.

[16] J. H. Wu, X. Zhai, and Z. M. Huang, "Incentives for information sharing in duopoly with capacity constraints," OMEGA - The International Journal of Management Science, vol. 36, no. 6, pp. 963-975, 2008.

[17] K. Natarajan, D. Kostamis, and A. Merserean, "Strategic demand information sharing between competitors," Working Paper, University of North Carolina, Chapel Hill, NC, USA, 2013.

[18] L. Jiang and Z. Hao, "On the value of information sharing and cooperative price setting," Operations Research Letters, vol. 42, no. 6-7, pp. 399-403, 2014.

[19] L. Li, "Information sharing in a supply chain with horizontal competition," Management Science, vol. 48, no. 9, pp. 1196-1212, 2002.

[20] H. Zhang, "Vertical information exchange in a supply chain with duopoly retailers," Production Engineering Research and Development, vol. 11, no. 4, pp. 531-546, 2002.

[21] K. S. Anand and M. Goyal, "Strategic information management under leakage in a supply chain," Management Science, vol. 55, no. 3, pp. 438-452, 2009.

[22] G. Kong, S. Rajagopalan, and H. Zhang, "Revenue sharing and information leakage in a supply chain," Management Science, vol. 59, no. 3, pp. 556-572, 2013.

[23] N. Shamir, "Cartel formation through strategic information leakage in a distribution channel," Marketing Science, vol. 36, no. 1, pp. 70-88, 2017. 
[24] A. Y. Ha, Q. Tian, and S. Tong, "Information sharing in competing supply chains with production cost reduction," Manufacturing \& Service Operations Management, vol. 19, no. 2, pp. 246-262, 2017.

[25] A. Y. Ha, S. Tong, and H. Zhang, "Sharing demand information in competing supply chains with production diseconomies," Management Science, vol. 57, no. 3, pp. 566-581, 2011.

[26] G. D. Wu, Q. S. Kong, J. G. Shi, H. R. Karimi, and W. Zhang, "Information sharing and channel construction of supply chain under asymmetric demand information," Journal of Applied Mathematics, vol. 2014, Article ID 107589, 8 pages, 2014.

[27] L. Jiang and Z. Hao, "Incentive-driven information dissemination in two-tier supply chains," Manufacturing and Service Operations Management, vol. 18, no. 3, pp. 393-413, 2016.

[28] M. Zhou, B. Dan, S. Ma, and X. Zhang, "Supply chain coordination with information sharing: the informational advantage of GPOs," European Journal of Operational Research, vol. 256, no. 3, pp. 785-802, 2017.

[29] P. Zhang and Z. Xiong, "Information sharing in a closed-loop supply chain with asymmetric demand forecasts," Mathematical Problems in Engineering, vol. 2017, Article ID 9785759, 12 pages, 2017.

[30] D.-Q. Yao, X. H. Yue, and J. Liu, "Vertical cost information sharing in a supply chain with value-adding retailers," Omega , vol. 36, no. 5, pp. 838-851, 2008.

[31] H. Liu, S. Sun, M. Lei, G. K. Leong, and H. Deng, "Research on cost information sharing and channel choice in a dual-channel supply chain," Mathematical Problems in Engineering, vol. 2016, Article ID 4368326, 12 pages, 2016.

[32] D. Kostamis and I. Duenyas, "Purchasing under asymmetric demand and cost information: when is more private information better?" Operations Research, vol. 59, no. 4, pp. 914-928, 2011.

[33] G. P. Cachon and M. A. Lariviere, "Contracting to assure supply: how to share demand forecasts in a supply chain," Management Science, vol. 47, no. 5, pp. 629-646, 2001.

[34] C. Eksoz, S. A. Mansouri, and M. Bourlakis, "Collaborative forecasting in the food supply chain: a conceptual framework," International Journal of Production Economics, vol. 158, pp. 120135, 2014.

[35] M. A. Resende-Filho and T. M. Hurley, "Information asymmetry and traceability incentives for food safety," International Journal of Production Economics, vol. 139, no. 2, pp. 596-603, 2012.

[36] C. A. Yano and H. L. Lee, "Lot sizing with random yields: a review," Operations Research, vol. 43, no. 2, pp. 311-334, 1995.

[37] Y.-J. Chen and C. S. Tang, "The economic value of market information for farmers in developing economies," Production Engineering Research and Development, vol. 24, no. 9, pp. 14411452, 2015.

[38] C. S. Tang, Y. Wang, and M. Zhao, "The implications of utilizing market information and adopting agricultural advice for farmers in developing economies," Production Engineering Research and Development, vol. 24, no. 8, pp. 1197-1215, 2015.

[39] S. Deo and C. J. Corbett, "Cournot competition under yield uncertainty: the case of the U.S. influenza vaccine market," Manufacturing and Service Operations Management, vol. 11, no. 4, pp. 563-576, 2009.

[40] S. Y. Tang and P. Kouvelis, "Supplier diversification strategies in the presence of yield uncertainty and buyer competition," Manufacturing and Service Operations Management, vol. 13, no. 4, pp. 439-451, 2011. 

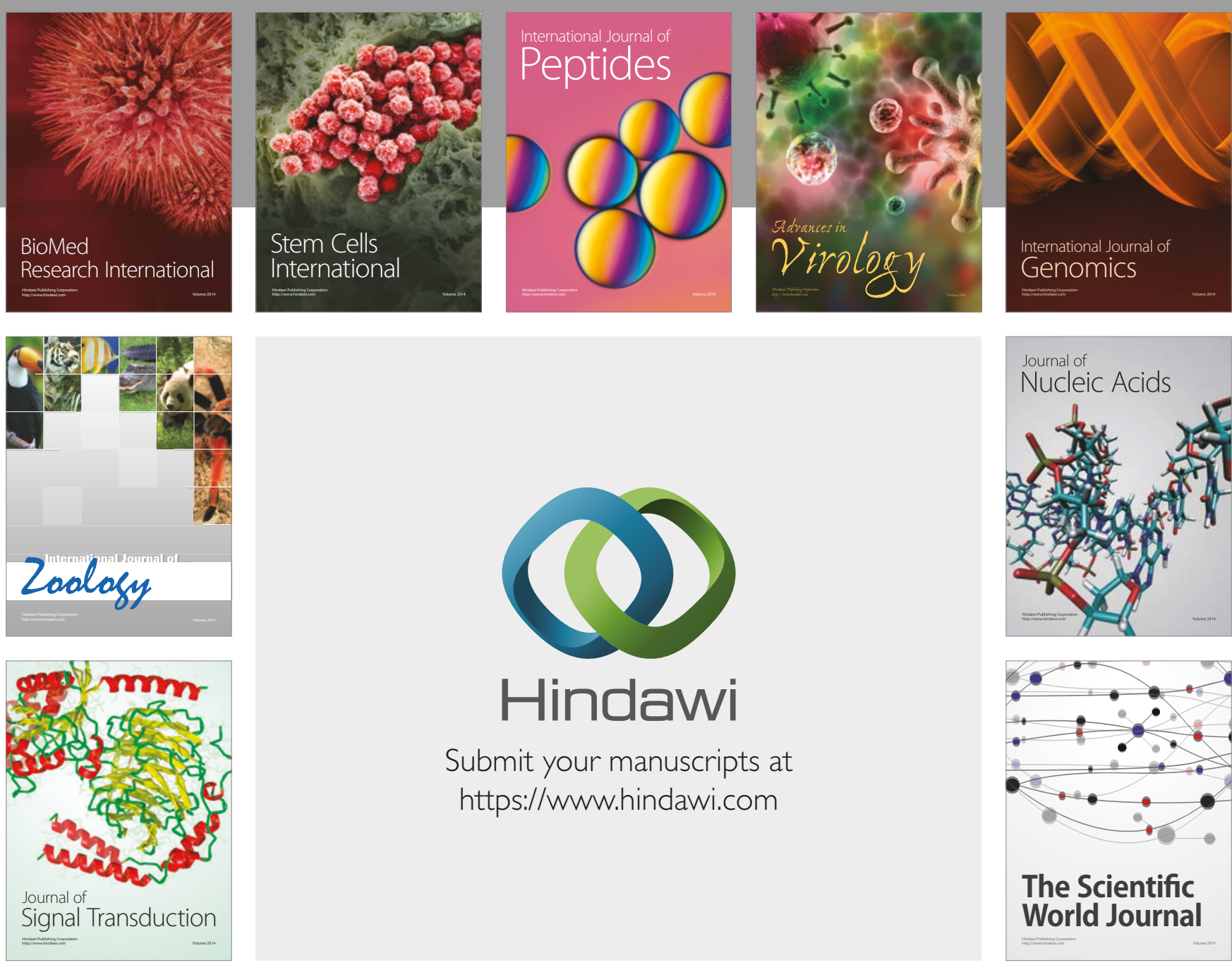

Submit your manuscripts at

https://www.hindawi.com
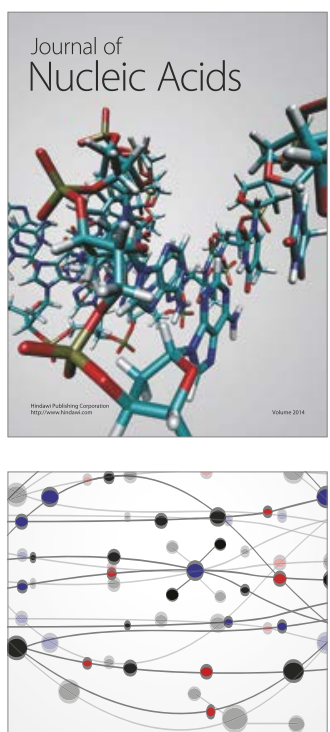

The Scientific World Journal

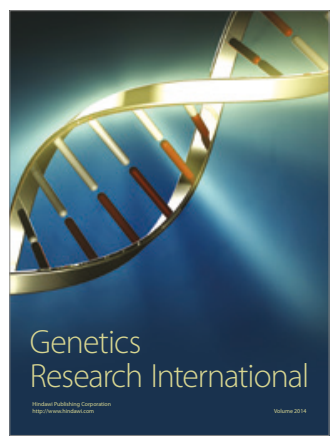

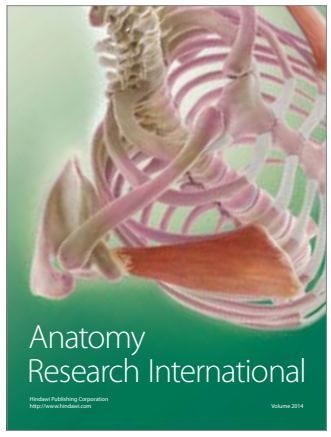

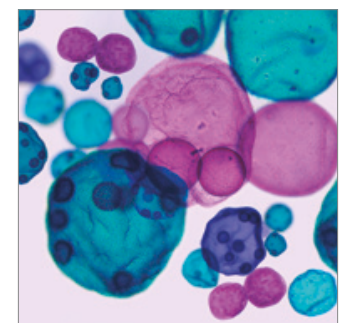

International Journal of Microbiology
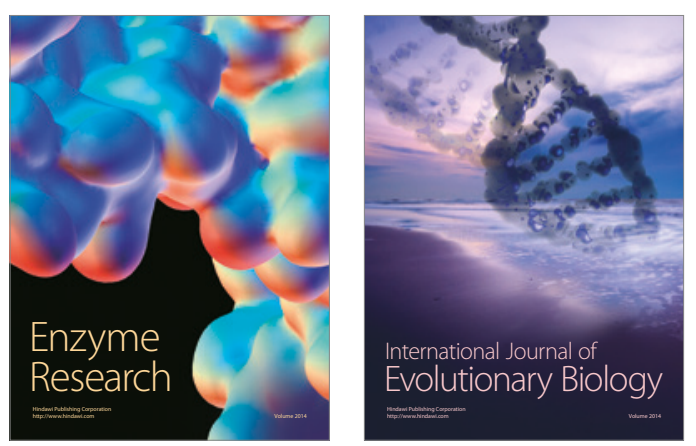
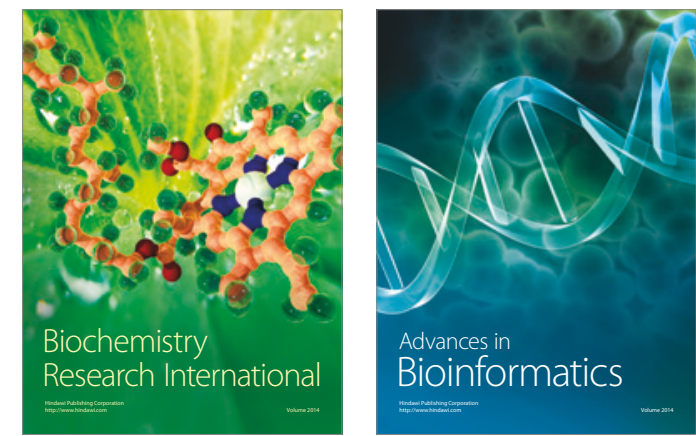

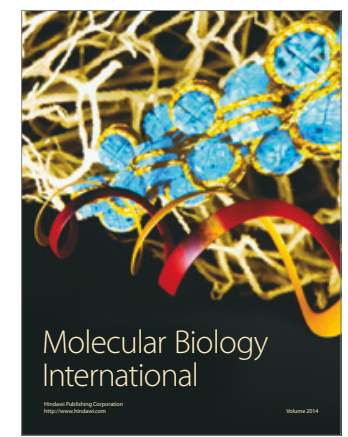

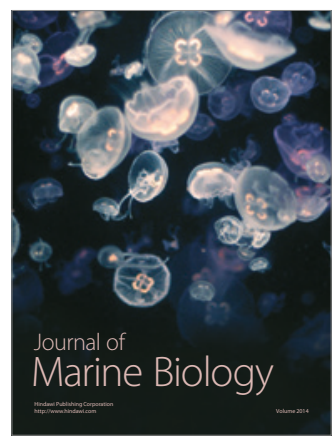

